\title{
Positron Annihilation Studies of Mesoporous Iron-Molybdenum Modified MCM-41 Silica
}

\author{
Z. Surowiec ${ }^{a, *}$, M. Wiertel ${ }^{a}$, W. GAC ${ }^{b}$ AND M. BudzyŃski ${ }^{a}$ \\ ${ }^{a}$ Institute of Physics, M. Curie-Skłodowska University, pl. M. Curie-Skłodowskiej 1, 20-031 Lublin, Poland \\ ${ }^{b}$ Faculty of Chemistry, M. Curie-Skłodowska University, pl. M. Curie-Skłodowskiej 1, 20-031 Lublin, Poland \\ Iron-molybdenum modified MCM-41 mesoporous materials were obtained by the application of direct hy- \\ drothermal method. An incorporation of iron and molybdenum ions in the synthesis stage led to structural \\ changes of the MCM-41 support. With an increasing metals content, cylindrical pores of silica are destroyed. \\ X-ray diffraction investigations indicated that both iron and molybdenum ions were first embedded in the silica \\ walls and then formed highly dispersed species in the pores. The porous structure of the studied samples was \\ examined by means of $\mathrm{N}_{2}$ adsorption/desorption and PALS methods. The longest-lived $o$-Ps lifetime component \\ remains constant but its intensity monotonically decreases, except for the sample with the highest metals content. \\ These results indicate that an effect of $o$-Ps formation inhibition occurs.
}

DOI: 10.12693 /APhysPolA.125.789

PACS: 34.80.Lx, 78.67.Bf, 78.70.Bj, 62.23.Pq, 78.67.Sc

\section{Introduction}

MCM-41 materials belong to a family of mesoporous silicates (M41S) which was discovered by Mobil Oil researchers [1]. The materials consist of hexagonal arrays of uniform sized cylindrical pores with diameters changing from 2-10 nm depending on a method of preparation. The channel pores are created by using the self-assembly of silicates with surfactant as the templates and followed by calcination of organic part. The material is characterized by large specific surface area often exceeding $1000 \mathrm{~m}^{2} / \mathrm{g}$. However, pure MCM41 shows very limited catalytic activity due to the lack of lattice defect and redox properties. Hence, it requires modification to introduce other features into mesoporous silicates to improve their properties. Modification of MCM-41 surface can improve the catalytic activity by raising their hydrophobicity [2]. The introducing of metal ions onto mesoporous silicates leads to increase of active sites quantity and improves the catalytic activity. Many transition metals such as $\mathrm{Ti}, \mathrm{Pt}, \mathrm{Fe}, \mathrm{Ni}$ and others have been incorporated into the wall structure to obtain the mesoporous materials with catalytic properties especially in the selective oxidation, epoxidation, alkylation, and isomerisation of organic compounds [3-7]. Iron-molybdenum oxide systems seem to have particularly interesting catalytic properties. They have found wide applications as catalysts in the reactions of selective oxidations, mainly methanol to formaldehyde [8]. The decrease of activity and increase of selectivity relative to the total oxidation products have been ascribed to the transformation of the structure of catalysts, related with the formation of volatile molybdenum oxide and iron-rich surface oxide phases [9].

*corresponding author; e-mail: zbigniew.surowiec@umcs .pl

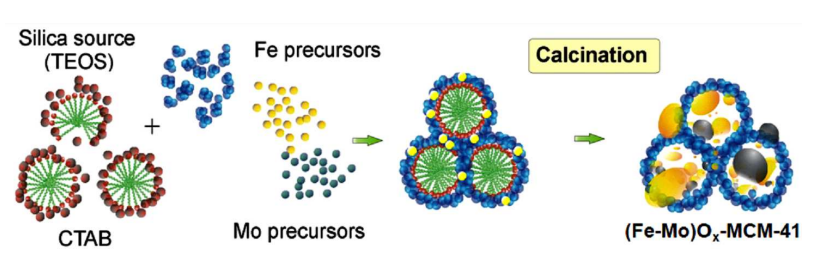

Fig. 1. The synthesis stages of high surface area oxide nanomaterials by means of direct hydrothermal method (DHM).

One of the methods of the modification of the MCM- 41 structure is the direct hydrothermal synthesis in which the metal to be incorporated is directly added to the synthesis mixture. Schematic picture of this route is shown in Fig. 1. In this method the silica source, the surfactant and the metal precursor are allowed to react with each other in a certain sequence to form a gel. The resulting gel is autoclaved in an etuve for the hydrothermal synthesis stage. During the synthesis, the MCM-41 crystals form and precipitate. As a result of the direct hydrothermal synthesis, a relatively homogeneous incorporation of the desired metal into the walls of the MCM-41 is achieved and a catalyst with successful redox properties is obtained [10].

The aim of the presented studies was preparation and investigation of the structural properties of ironmolybdenum modified MCM-41 silica.

\section{Experimental}

Iron-molybdenum silica mesoporous materials were obtained by the application of direct hydrothermal method described in detail in [11].

Molar ratio of iron to molybdenum $\mathrm{Fe} / \mathrm{Mo}$ was the same in all samples and equal to 2:3. The introduced 
amounts of iron were varied, in order to obtain samples containing: 3 wt $\%$ Fe (denoted as Fe-Mo(A)-Si), $6.5 \mathrm{wt} \% \mathrm{Fe}$ (denoted as $\mathrm{Fe}-\mathrm{Mo}(\mathrm{B})-\mathrm{Si}$ ), $12.5 \mathrm{wt} \% \mathrm{Fe}$ (denoted as $\mathrm{Fe}-\mathrm{Mo}(\mathrm{C})-\mathrm{Si}$ ) and 24.3 wt\% $\mathrm{Fe}$ (denoted as $\mathrm{Fe}-\mathrm{Mo}(\mathrm{D})-\mathrm{Si})$.

The porous structure of materials was determined by the analysis of the nitrogen adsorption/desorption isotherms obtained volumetrically at $77 \mathrm{~K}$ using ASAP $2405 \mathrm{~N}$ apparatus. Before measurements, the samples were outgassed (under $\approx 10^{-2} \mathrm{~Pa}$ ) at $200^{\circ} \mathrm{C}$. The adsorption data were used to evaluate the Brunauer-EmmettTeller (BET) specific surface area, $S_{\mathrm{BET}}$ (from the linear BET plots).

The mesostructural and ordered characteristics of the samples were determined by low angle XRD method. In this study, the XRD patterns of the ironmolybdenum modified MCM-41 silica are obtained using a EMPYREAN diffractometer system with a $\mathrm{Cu} K_{\alpha}$ radiation source. The scanning of the samples is started from the low $2 \theta$ value of $0.6^{\circ}$ in order to obtain the low angle peaks characteristic of the MCM-41 structure.

In positron annihilation lifetime spectroscopy (PALS) measurements, the ${ }^{22} \mathrm{Na}$ positron source sealed in a kapton envelope was placed between two layers of the powder sample put in a plastic container. Each layer was $3 \mathrm{~mm}$ thick. This thickness is enough to stop all the positrons.

Positron lifetime spectra were recorded at room temperature using a fast-slow delayed coincidence spectrometer with pulse pile-up inspection. The spectrometer was equipped with two scintillation counters consisting of $\mathrm{BaF}_{2}$ coupled to XP2020Q photomultiplier tubes. In porous media, an essential number of $o$-Ps atoms decay into 3 gamma-quanta. Their energy spectrum is continuous, extending from 0 to $511 \mathrm{keV}$. Thus, to improve the efficiency of counting the stop energy window in the spectrometer was widely open ( $80 \%$ of the energy range). At such a setting the resolution time was $0.29 \mathrm{~ns}$ (for ${ }^{60} \mathrm{Co}$ source with a ${ }^{22} \mathrm{Na}$ window). Total counts number in each spectrum was equal to at least ten million. The time base of the PALS setup was $1 \mu \mathrm{s}$ (8192 channels). The positron lifetime spectra were analyzed using the LT program [12].

\section{Results and discussion}

As MCM-41 consists of a hexagonal array of channels thus XRD can be used to characterize the material structurally although it has amorphous walls. This method can provide an independent estimate of pore sizes.

The low-angle XRD patterns of FeMo-MCM-41 with different weight ratios FeMo:Si are shown in Fig. 2a. The Bragg reflections obtained in the low angle range for samples FeMo-MCM-41(A) and FeMo-MCM-41(B) are known to be characteristic of the MCM- 41 structure. The three peaks visible in XRD pattern related to hexagonal array of parallel silica tubes, correspond to (100), (110), and (200) planes. When the metals to silica ratio increases the peaks intensities were decreased. The (100)

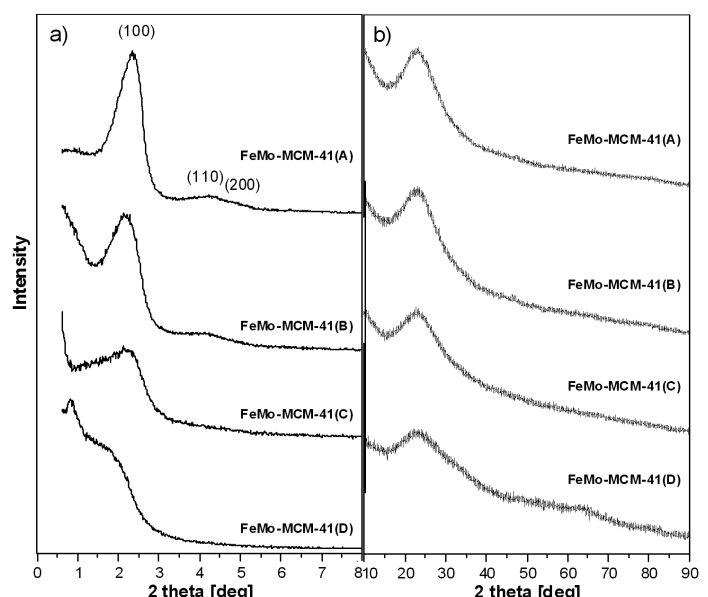

Fig. 2. XRD diffraction patterns at low (a) and high (b) angles of samples $\mathrm{FeMo}(\mathrm{A})-\mathrm{Si}, \mathrm{FeMo}(\mathrm{B})-\mathrm{Si}$, $\mathrm{FeMo}(\mathrm{C})-\mathrm{Si}$, and $\mathrm{FeMo}(\mathrm{D})-\mathrm{Si}$.

peak at $2 \theta$ equal to $2.33^{\circ}$ for sample FeMo-MCM-41(A) was shifted to the lower diffraction angle $2 \theta$ equal to $2.20^{\circ}$ for sample FeMo-MCM-41(B). This result is due to the incorporation of iron and molybdenum atoms into the mesostructure.

The characteristic lattice parameter $a$ (the repeating distance between two pore centres) can be calculated according to Eq. (1):

$$
a=\frac{2}{\sqrt{3}} d_{(100)} \text {. }
$$

The pore wall thickness $\delta$ was then estimated from the average pore diameter $\left(d_{\mathrm{p}}\right)$ and the lattice parameter $(a)$ using Eq. (2):

$$
\delta=a-0.95 d_{\mathrm{p}} .
$$

For FeMo-MCM-41(C), only a broadened (100) peak is observable, indicating the formation of disordered mesostructures. Therefore, with increase of iron molybdenum loading, ordering of mesostructures over long range gets worse. For the sample with 32.5 wt\% metals content, the diffraction peak corresponding to (100) plane of MCM-41 take form of a hump. It suggests that the hexagonal pore structure of MCM-41 is distorted.

The XRD measurements in a wide range of angles were carried out in order to obtain information about formation of crystallites in the mesoporous silica template. As shown in Fig. 2b, all studied samples exhibit a broadened diffraction peak centred around $2 \theta$ equal to $23^{\circ}$ due to the amorphous silica. The lack of structural diffraction peaks indicate that iron-molybdenum species are strongly dispersed in the template. This result is in accordance with the Mössbauer data [13].

In Fig. 3 pore sizes distributions (PSDs) obtained by means of the Barrett-Joyner-Halenda (BJH) method are shown. The average pore diameters $d_{\mathrm{p}}$ of the samples were determined from peaks positions in mesoporous range. 


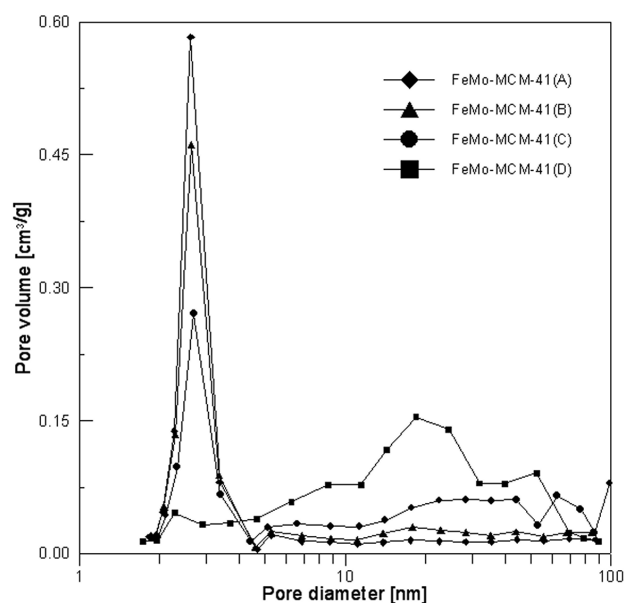

Fig. 3. Pore size distributions determined from adsorption/desorption isotherms of the investigated samples by the BJH method.

All samples, except FeMo-MCM-41(D), have narrow pore size distributions. In the sample with the highest metals content very wide PSD without noticeable maximum occurs. Also contribution of macropores of sizes above $10 \mathrm{~nm}$ is significant.

The chemical compositions, lattice parameters, and the results of $\mathrm{N}_{2}$ (adsorption/desorption) A/D measurements analysis are summarized in Table I. From the collected data it is seen that pore wall thicknesses increase with the incorporation of metal ions to silica matrices.

TABLE I

Physical properties of FeMo modified MCM-41 samples.

\begin{tabular}{c|c|c|c|c|c|c|c}
\hline \hline $\begin{array}{c}\text { Sample } \\
\begin{array}{c}\text { FeMo- } \\
\text {-MCM- }\end{array}\end{array}$ & $\begin{array}{c}\text { Metal con- } \\
- \text { centration } \\
\text { [wt\%] }\end{array}$ & $d(100)$ & $\begin{array}{c}\text { Lattice } \\
\text { parameter } \\
a[\mathrm{~nm}]\end{array}$ & $\begin{array}{c}S_{\mathrm{BET}} \\
{\left[\mathrm{m}^{2} / \mathrm{g}\right]}\end{array}$ & $\begin{array}{c}\text { Pore } \\
\text { diameter } \\
d_{\mathrm{p}}[\mathrm{nm}]\end{array}$ & $\begin{array}{c}\text { Pore wall } \\
\text { thickness } \\
\delta[\mathrm{nm}]\end{array}$ \\
\cline { 2 - 7 } (A) & 3 & 1.1 & 37 & 4.27 & 1133.0 & 2.62 & 1.78 \\
(B) & 6.5 & 2.2 & 40 & 4.62 & 1051.4 & 2.63 & 2.12 \\
(C) & 12.5 & 4.2 & 40 & 4.62 & 817.7 & 2.69 & 2.18 \\
(D) & 24.3 & 8.2 & uncertain & uncertain & 472.9 & uncertain & uncertain
\end{tabular}

In order to determine the porosity structure of FeMo modified MCM-41 structure PALS method was used. The obtained PALS spectra are shown in Fig. 4.

In the PALS spectra five-components were assumed. The first two very short-lived components are related to intrinsic decay of $p$-Ps atoms $\left(\tau_{1} \approx 0.125 \mathrm{~ns}\right)$ and annihilation of free positrons $\left(\mathrm{e}^{+}, \tau_{2} \approx 0.40 \mathrm{~ns}\right)$, respectively. Free positrons annihilation takes place in vacancy clusters formed in iron-molybdenum oxide nanoparticles or on their surfaces [14] and in small structural cages in amorphous silica walls, for instance tetrahedrally coordinated $\mathrm{Si} / \mathrm{Fe}$ or $\mathrm{Si} / \mathrm{Mo}$ monovacancies in $\left[\mathrm{V}_{\mathrm{Si} / \mathrm{Fe}} \mathrm{O}_{4 / 2}\right]$ - basic structural blocks. The $\tau_{1}$ parameter was fixed in the fitting procedure in order to obtain reasonable standard deviations for the parameters of long-lived $o$-Ps components. The $\tau_{2}$ lifetime value remains constant but its intensities change from about $40 \%$ in pure MCM-41

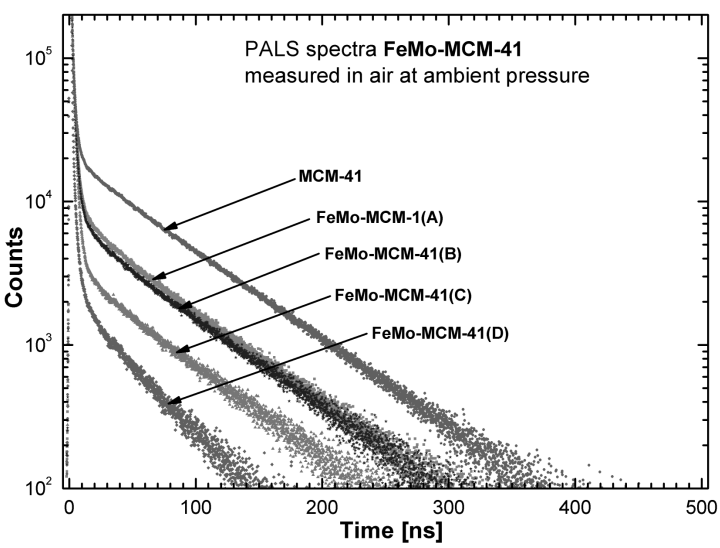

Fig. 4. Positron annihilation lifetime spectra of four FeMo-Si samples measured in air, normalized to time of measurement.

to $64 \%$ in the sample with maximum metals. It can be explained as follows. Along with an increase of Fe and Mo content the numbers of atoms in silica framework sites and amount of intermetallics in the form of ironmolybdenum oxide nanocrystallites deposited mainly on surfaces of MCM-41 walls increases. As a result of interaction between free positrons and modified silica walls and nanocrystallites relative contribution of free annihilation component $I_{2}$ to total annihilation process significantly augments due to increasing probability of free positron annihilation in media enriched in excess electrons.

TABLE II

The lifetimes and intensities of $o$-Ps components measured in air for FeMo modified MCM-41 samples.

\begin{tabular}{c|c|c|c|c|c|c}
\hline \hline $\begin{array}{c}\text { Sample } \\
\text { FeMo- } \\
\text {-MCM-41 }\end{array}$ & $\tau_{3}$ & $\tau_{4}$ & $\tau_{5}$ & $I_{3}$ & $I_{4}$ & $I_{5}$ \\
\cline { 2 - 7 } & \multicolumn{3}{|c|}{$[\mathrm{ns}]$} & & \multicolumn{3}{|c}{$[\%]$} \\
\hline PCM-41 & $1.59(10)$ & $18.39(75)$ & $71.06(30)$ & $10.73(27)$ & $1.00(14)$ & $16.67(28)$ \\
\hline (A) & $1.56(9)$ & $14.89(49)$ & $69.38(29)$ & $12.22(26)$ & $0.87(8)$ & $5.96(16)$ \\
(B) & $1.55(9)$ & $13.49(53)$ & $69.72(39)$ & $11.16(27)$ & $0.83(10)$ & $5.66(16)$ \\
(C) & $1.53(10)$ & $8.68(55)$ & $66.22(46)$ & $11.03(28)$ & $0.46(12)$ & $2.75(12)$ \\
(D) & $0.83(13)$ & $4.79(38)$ & $45.53(46)$ & $5.61(47)$ & $0.48(14)$ & $1.16(8)$
\end{tabular}

Moreover, in the investigated samples of FeMo-MCM-41 the three long-lived o-Ps components were observed in the positron lifetime spectra. Ortho-positronium annihilation process parameters values are given in Table II. The first of them $\tau_{3} \approx 1.5 \mathrm{~ns}$ probably arises due to the pick-off annihilation of $o$-Ps formed in the some kind of open-volume defects on the surfaces of amorphous silica walls of the order of $0.26 \mathrm{~nm} \mathrm{[15].} \mathrm{The} \mathrm{medium-lived}$ component $\left(\tau_{4}\right)$ results from decay of $o$-Ps trapped inside of nanochannels of MCM-41 silica. The lifetimes of this component decrease significantly only for the last two samples. The positronium atoms confined in such pores types are more and more effectively quenched by pick-off process because of decreasing their free volumes.

It seems that these lifetimes are generally too short in comparison to those expected for nanochannel diameters 
obtained from $\mathrm{N}_{2}$ adsorption/desorption measurement (see Table I). The chemical quenching on internal silica walls surfaces modified by $\mathrm{Fe}$ and Mo species is a main reason for too low $\tau_{4}$ values. The longest-lived component $\left(\tau_{5}\right)$ is related to 3 -gamma annihilation of $o$-Ps forming in intergranular spaces of the material, with some contribution from pick-off annihilation. The intensities of the longest-lived component $\left(I_{5}\right)$ measured in air systematically decrease along with an increase of FeMo content in the samples. For the sample FeMo-MCM-41(D) also shortening of the longest-lived component lifetime is observed. For the first three samples these effects are only a result of chemical $o$-Ps formation inhibition. In the fourth sample also quenching effect occurs.

For the metals content up to $8.7 \mathrm{wt} \%$, the lifetimes of all three $o$-Ps components remain practically unchanged. This result is in accordance with nitrogen adsorption/ desorption measurements in which the pore sizes are constant. Introduction of the Fe and Mo atoms into silica source in the synthesis state in the given quantity do not modify the MCM-41 mesoporous structure. The increase of metals content is reflected in $I_{4}$ and $I_{5}$ intensities values. The lifetimes and intensities of $o-P s$ components are significantly changed in the samples with higher metals addition. The decrease of intensities is affected by effect of positronium inhibition. In this case, the mechanism of inhibitions depends on positron free annihilation in metal clusters which are formed at higher metal concentration.

\section{Conclusions}

An introduction of different amounts of iron and molybdenum to the synthesis mixture of the mesoporous silica by means of direct hydrothermal led to the modification of the structural and surface properties of materials. All methods applied detect particularly considerable changes for FeMo exceeding 16.7 wt\%.

However, in contrary to empty mesoporous support where the PALS method allows for quantitative estimating pore and free volume sizes, in the modified FeMo
MCM-41 silica it is too difficult to be used. The application of PALS method in such complex materials requires developing more sophisticated models able to take into account chemical quenching and inhibition of $\mathrm{o}-\mathrm{Ps}$ formation.

\section{References}

[1] C.T. Kresge, M.E. Leonowicz, W.J. Roth, J.C. Vartuli, J.S. Beck, Nature 359, 710 (1992).

[2] R.S. Robert, A. Rafael, A.D. James, W.R. Thatcher, Micropor. Mesopor Mater. 66, 53 (2003).

[3] A. Taguchi, F. Schüth, Micropor. Mesopor. Mater. 77, 1 (2005).

[4] D. Liu, W.N. Evelyn Cheo, Y.W. Yvonne Lim, A. Borgna, R. Lau, Y. Yang, Catal. Today 154 , 229 (2010).

[5] Z. Surowiec, M. Wiertel, M. Budzyński, W. Gac, Nukleonika 58, 87 (2013).

[6] Z. Surowiec, M. Wiertel, M. Budzyński, J. Sarzyński, J. Goworek, J. Non-Cryst. Solids 354, 4271 (2008).

[7] I. Alonso-Lemu, Y. Verde-Gómez, L. ÂlvarezContreras, Int. J. Electrochem. Sci. 6, 4176 (2011).

[8] A.P.V. Soares, M.F. Portela, A. Kiennemann, Catal. Rev. Sci. Eng. 47, 125 (2004).

[9] G. Centi, F. Fazzini, J.L.G. Fierro, M.L. Granados, R. Sanz, D. Serrano, Stud. Surf. Sci. Catal. 118, 577 (1998).

[10] K.J. Chao, C.N. Wu, H. Chang, L.J. Lee, S. Hu, J. Phys. Chem. B 101, 6341 (1997).

[11] Z. Surowiec, M. Wiertel, A. Marynowska, W. Gac, W. Zawadzki, Nukleonika 58, 137 (2013).

[12] J. Kansy, Nucl. Instrum. Meth. Phys. Res. A 374, 235 (1996).

[13] M. Wiertel, Z. Surowiec, M. Budzyński, W. Gac, Nukleonika 58, 245 (2013).

[14] S. Chakrabarti, S. Chaudhuri, P.M.G. Nambissan, Phys. Rev. B 71, 64105 (2005).

[15] S. Dannefaer, T. Bretagnon, D. Kerr, J. Appl. Phys. 74, 884 (1993). 